Review Article

www.ijrap.net

\title{
ETHNOPHARMACOLOGY, PHYTOCHEMISTRY AND BIOLOGICAL ACTIVITY OF INULA RACEMOSA HOOK. F: A REVIEW
}

Qurba Firdous ${ }^{1}$, Mohammad Faizan Bhat ${ }^{2}$, Mubashir Hussain $\mathrm{M}^{3 *}$

${ }^{1}$ Department of Pharmaceutical Sciences, University of Kashmir, Srinagar, J\&K, India

${ }^{2}$ Research Fellow, Department of Pharmaceutical Sciences, University of Kashmir, Srinagar, J\&K, India

${ }^{3}$ Sr. Assistant Professor, Department of Pharmaceutical Sciences, University of Kashmir, Srinagar, J\&K, India

Received on: 25/10/17 Accepted on: 18/12/17

*Corresponding author

E-mail: mubashir@kashmiruniversity.ac.in

DOI: 10.7897/2277-4343.09120

\begin{abstract}
Inula racemosa Hook. F (Asteraceae) commonly known as Pushkaramula is a well documented Indian medicinal plant. Pushkarmula is one of the herbs mentioned in all Ayurvedic scriptures. It possesses various synonyms like kasari- an enemy of cough, sulahara - pain killer, sughandhika fragrant etc. The great sage Charaka has categorized it as hikkanigrahana - stops hiccup and svasahara - alleviates the breathlessness, asthma. It is also best medicament for pleurisy along with cough and asthma. Pushkarmula is highly acclaimed to be the drug of choice for pleurisy (parsvasula). It has also anti-inflammatory, cardiovascular, hypoglycemic, antianginal, analgesic and antibacterial properties. Many of these ethnomedicinal properties have been experimentally proven in different animal models. This article is an attempt to collect and review all the data concerning systemic scientific study of ethnopharmacology of I. racemosa, its isolated phytoconstituents and bioactivity of extracts as well as isolated compounds from the plant.
\end{abstract}

Keywords: Inula racemosa, ethnopharmacology, phytochemistry, bioactivity.

\section{INTRODUCTION}

The plant, Inula racemosa is abundantly found in India, China and Europe. The plant grows in temperate and alpine Western Himalayas from 1300 to 4500 meters elevation. The plant is distributed in temperate alpine Himalayas at an altitude of 1,500- 4,200m from Kashmir to Kumaon, Afghanistan to Central Nepal. It occurs wild among strong alpine scrub vegetation in the cold arid habitat of NW Himalayas between 2,700-3,500 $\mathrm{m}$ in the eastern Ladakh (Leh) region of Kashmir.

Domesticated forms of this incipient cultigen are cultivated on borders of agricultural fields of wheat, barley and buckwheat both in Kashmir and Lahaul valley of Himachal Pradesh ${ }^{1}$. The plant is a stout shrub, bearing large leaves arranged in a racemose manner. The stem is grooved and all vegetative parts are scabridtomentose. Lower leaves are narrowed to a winged leaf stack. Upper leaves are lanceolate and stem clasping. The abaxiallaminal face is densely tomentose. The fresh root is brown and becomes grayish on drying. The fresh roots resemble the aroma of camphor. The fruits, slender achenes have long pappus hairs. Root stock is branched. Sometimes a number of roots are found in the collar zone, though usually few occur in each clump. These roots have a dull brownish skin with yellowish colour inside. They possess a sweet and somewhat camphoraceous odour and have a bitter taste ${ }^{2}$.

Inula racemosa is known to be used in traditional medicine throughout the world, especially East Asia and Europe. Apart from being used for other ailments, the plant extract and its isolated active constituents show promising activity against abdominal pain, acute enteritis, bacillary dysentery, expectorant and tonic ${ }^{2}$. Inula racemosa is also used in combination with other plant extracts and used for various conditions including hyperlipidemia, angina and patients with Ischemic Heart
Disease $^{3}$. Various active constituents have been isolated from the plant, most important being sesquiterpene lactonesAlantolactone (ALT), and isoalantolactone (IALT) that show anti-inflammatory and decreased proteolytic activity ${ }^{4-5}$. This review is therefore aimed to comprehensively collect all the literature regarding ethnopharmacology, phytoconstituents, and biological activity of Inula racemosa.

\section{Ethnopharmacology}

Inula racemosa has been used as traditional medicine in East Asia and Europe. In China it has been prescribed for abdominal pain, acute enteritis and bacillary dysentery. The roots are widely used as indigenous medicine, as an expectorant and in veterinary medicine as a tonic ${ }^{2}$. Native Americans use this plant for treatment of tuberculosis ${ }^{6}$. Root powder is reportedly hypoglycemic and hypocholesterolemic in human subjects ${ }^{7}$. It brought about a beneficial improvement in ST-T changes in ECG of patients with Ischemic heart disease (IHD) ${ }^{8}$.

Combination therapy of Inula racemosa with other plants and extracts has also shown substantial biological activities. It is anti-anginal and hypolipidemic when used in combination with guggulu in patients with Ischemic heart disease ${ }^{3}$.It exerts cardioprotective effect in isoproterenol induced myocardial ischemia in rats when used in combination with drugs Terminalia arjuna and Commiphora mukul ${ }^{9}$.It reduced corticosteroid induced hyperglycaemia in mice when used with Gymnema leaf extract ${ }^{10}$.

The drug exhibited negative chronotropic effect and positive ionotropic effect on isolated frog heart with petroleum ether extract $(200 \mathrm{mg} / \mathrm{kg})$.Further, increase of dose to $400 \mathrm{mg} / \mathrm{kg}$ exhibited adrenaline-induced beta blocking activity in rats ${ }^{11}$. Petroleum ether extract exhibited less hepatoprotective activity 
as compared to the aqueous, methanolic and total aqueous extract ${ }^{12}$.Moreover, with the alcoholic extract significant protection against egg albumin induced passive cutaneous anaphylaxis was provided and the alcoholic extract is non-toxic upto $2100 \pm 60 \mathrm{mg} / \mathrm{kg}$ i.p in rats ${ }^{13}$. Furthermore, antidermatophytic and anti-cholinergic activities were exhibited by the crude alcoholic extract, the former reportedly localized in the hexane soluble fraction ${ }^{14}$.

\section{Isolated phytochemical constituents from Inula racemosa}

Inula racemosa yields large amounts of sesquiterpene lactones as-Alantolactone (ALT) and isoalantolactone (IALT) ${ }^{4}$, Dihydroalantolactone, dihydroisoalantolactone, inunolide ${ }^{15}$, dihydroinunolide, neoalantolactone, isoalloalantolactone ${ }^{16}$, alloalantolactone ${ }^{17}$, inunal, isoinunal ${ }^{18}$, alantodiene and isoalantodiene ${ }^{19}$ are other sesquiterpene lactones isolated from the non polar fractions of the root.

Daucosterol, D- mannitol and Beta sitosterol have also been reported in good quantities from the roots ${ }^{20}$. Roots of 'mano' from Kashmir is reported to yield 5.7-6.2\% petroleum ether extract while those from Lahaul valley, Himachal Pradesh reportedly yield $8.5 \% \mathrm{w} / \mathrm{w}$ constituted of $83 \%$ lactones $^{1}$. The major lactones ALT and IALT are in the ratio 4:6.

Investigation on the aerial parts of Inula racemosa reported the presence of several other sesquiterpene lactones namely ivalin acetate, 2d-OH alantolactone, 1- desoxy-8-epi-ivangustin, 8-epiisoivangustin, $9 \beta-\mathrm{OH}$ costunolide, $9 \beta$-propionyloxycostunolide, $9 \beta$-(2-methylbutaryloxyl) costunolide, $4 \beta$-5 $\alpha$-epoxy- $10 \alpha, 14 \mathrm{H}$ inuviscolide, $4 \beta$, 5 $\alpha$-epoxy-4,5-cis-inunolide, 4H-tomentosin, $4 \mathrm{H}$ carbrone.

Structure ${ }^{21}$ was identified as 1-4-epi-alantolactone;(2) 4alpha,13-dihydroxy-5,7(11)-eudesmadien-12,8-olide.Six known eudesmane-12,8-olide,viz.,Septuplinolide(3)

22;macrophyllilactone E (4) ${ }^{23} ; 13$-acetyloxy-5,7(11)eudesmadien-12,8-olide (5) ${ }^{24} ; 11$ alpha,13-dihydro-2alphahydroxy-alantolactone (6) 25; 11,13-dihydroivalin (7) 26 ; Isoalantolactone $(\mathbf{8})^{27}$. (Figure 1)<smiles>C=C1C(=O)O[C@@H]2C[C@]3(C)C(=O)CC[C@@H](C)[C@]3(C)C[C@H]12</smiles><smiles>C[C@@]1(O)CCC[C@]2(C)C[C@H]3OC(=O)C(CO)=C3C=C12</smiles>
2

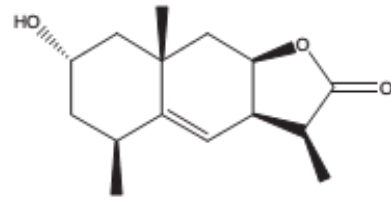

6<smiles>C=C1C(=O)O[C@@H]2C[C@@]3(C)CCC[C@](C)(O)C3C[C@H]12</smiles>
3<smiles></smiles>

7<smiles>C[C@H]1CCC[C@]2(C)C[C@H]3OC(=O)C(CO)=C3C=C12</smiles>

4<smiles>C[C@H]1CCC[C@]2(C)C[C@H]3OC(=O)C(CO)=C3C=C12</smiles>

5

Fig. 1. Structures of compounds 1-8.

\section{Biological Activities of Isolated Compounds}

"Sesquiterpene lactones are important because of their various biological activities and generic inhibition of enzymes ${ }^{28}$. They provide protection to the plant against various pathogenic organisms, insects and mammals. They are secondary metabolites of plant exerting various biochemical effects on other flora and vertebrate poisoning ${ }^{29}$.

The main sesquiterpene lactones found in Inula racemosa are ALT and IALT ${ }^{5}$.Amixture containing both the lactones is called Inula camphor (Helenin). In antiquity Inula helenium (Elecampane) contains both ALT and IALT. It was added to food as a seasoning in the middle age and later, it came to be used for medicinal purposes. Now a days, this mixture is the active principle of drug Alanton which is used for ulceration ${ }^{30}$. The drug is anti-inflammatory, antiproteolytic and is used to regulate the acidic function of the stomach. Alanton also promotes mucin formation and stimulates the regenerative capacity of the gastric mucosa ${ }^{5}$.
ALT and IALT promotes the growth in number of rootlets of Phaseolus aureus by a factor of 2-2.5 as compared to control in the experiment ${ }^{27}$. IALT is herbicidal because of its lipophilicity. It gets incorporated into cell membrane and does not reach to other regions of the plant ${ }^{31}$.

ALT and IALT showed an increase in the antioxidant activity of lipids at doses of $100-200 \mathrm{mgkg}^{-1}$, their action considerably more than antioxidant activity of $\alpha$-tocopherol and ubiquinone. The anti-tussive activity of helenin in guinea pigs has been seen, but it is half that of codeine ${ }^{5,32}$.

Both ALT and IALT possess antibacterial activities against many of gram positive and gram negative bacteria. ALT is completely inhibitory to Bacillus subtilis, while IALT exhibited weak antibacterial activity towards Bacillus subtilis and Bacillus vulgaris. ALT and helenin possessed pronounced inhibitory effect against Staphylococcus aureus and Mycobacterium tuberculosis at $31.2-62.5$ and $31.22 \mu \mathrm{g} / \mathrm{ml}$ respectively.

The antifungal activity has been studied in relation to more than 16 different cultures for both ALT and IALT, inhibited the 
growth of all the fungi studied, but the effects for each individual culture differed greatly. For zoophilic fungi Microsporium cookie and Trichophyton mentagrophytes both ALT and IALT exhibited their greatest inhibitory effect ${ }^{32}$.

The antibacterial and antifungal activity of sesquiterpene lactones has been mainly due to the presence or absence of $\alpha$ methylene group in the lactone ring alone ${ }^{28}$. This has been proved by the SAR of several sesquiterpene lactones.

Helenin was extracted from roots of Inula helenium. It consisted of $40 \%$ alantolactone and $60 \%$ isoalantolactone ${ }^{33}$. On investigation it was seen that the cytotoxic property of Helenin was mainly due to the presence of IALT in $\mathrm{it}^{34}$. By using the model of culture of human epidermal carcinoma cells, helenin was seen to possess IALT in it ${ }^{34}$. In vitro experiments of lines of human lung carcinoma cells also confirmed the cytotoxicities of ALT and IALT in $i^{35}{ }^{35}$ LD 50 was seen to be $4.6 \mu \mathrm{g} / \mathrm{ml}$ for ALT and $16 \mu \mathrm{g} / \mathrm{ml}$ for IALT. For complete suppression of growth of cells dose was $50 \mu \mathrm{g} / \mathrm{ml}$.

Biological activity of sesquiterpene lactones(ALT and IALT) is mainly due to the presence of reactive group $-\mathrm{CH}=\mathrm{C}-\mathrm{C}=\mathrm{O}$ in it.The basis of cytotoxic effect is due to Michael addition reaction between- $\mathrm{CH}=\mathrm{C}-\mathrm{C}=\mathrm{O}$ and the $\mathrm{SH}$ group of enzymes and proteins ${ }^{36}$. Lipophilicity also plays an important role in the biological activities of sesquiterpene lactones. With increase in lipophilicity cytotoxicity increases in Ivalin (2-OH alantolactone) and ivasperin (1, 2-dihydroxyalantolactone $)^{37}$.

The presence of $\alpha$-methylene- $\gamma$-lactone group is the reason for allergic contact dermatitis. The importance of reactivity of lactone has been shown in experiments because of interaction of ALT with various amino acids, due to which, ALT loses its immunological reactivity. Also ALT and 11, 13dihydroalantolactone is toxic in relation to the in vitroculture of leukocytes $^{38}$ (37)

\section{Biological Activity of Different extracts of Inula racemosa}

\section{Anti-Inflammatory Activity}

The anti-inflammatory activity of the ethanol extract of the roots of Inula racemosawas evaluated by carageenan-induced paw edema in rats. Ethanol extract showed maximum inhibition (34.17\%) at a dose of $200 \mathrm{mgkg}^{-1}$, body weight (b.w.) after $2 \mathrm{~h}$ of drug administration in carageenan-induced paw edema. Aspirin $\left(100 \mathrm{mgkg}^{-1}\right)$ was used as standard drug produced $17.50 \%$ of inhibition in paw edema ${ }^{39}$. In another study, aqueous extract of the roots of $I$. racemosa showed maximum inhibition(60\%) at a dose of $400 \mathrm{mgkg}^{-1}$ b.w. after $8 \mathrm{~h}$ of drug administration in carageenan-induced paw edema in rats, whereas standard drug indomethacin $\left(20 \mathrm{mgkg}^{-1}\right)$ produced $69 \%$ of inhibition ${ }^{40}$.

\section{Analgesic Activity}

Analgesic effect of ethanol extract of the roots of Inula racemosa was performed in albino rats of either sex using hot plate. Ethanol extract of the plant showed latency in percentage protection $(42.99 \%)$ at a dose of $200 \mathrm{mg} / \mathrm{kg}$, b.w. after $2 \mathrm{~h}$ of drug administration. Standard drug aspirin (100 mgkg-1) produced $65.47 \%$ latency of percentage protection ${ }^{41}$. Also, analgesic effect of aqueous extract of the roots of Inula racemose was performed in albino mice of either sex by acetic acid-induced writhing and tail immersion methods. Aqueous extract of plant at a dose of $400 \mathrm{mgkg}^{-1}$ showed higher latency of percentage protection $(63 \%)$, whereas in tail immersion model the highest enhanced reaction time was observed at 400 $\operatorname{mgkg}^{-1}(8.65 \pm 1.63 \text { at } 3 \mathrm{~h})^{41}$.

\section{Cytotoxic Activity}

In-vitro cytotoxic activity of $95 \%$ ethanol extract of Inula racemose roots and its different fractions (n-hexane, chloroform, n-butanol and aqueous) was evaluated on colon, ovary, prostate, lung, CNS and leukemia cancer cell lines using sulphorhodamine-B dye and MTT assay for HL-60 cell line. The major constituents of hexane fraction i.e. alantolactone and isoalantolactone was studied for its mode of action in HL-60 cells. The lowest $\mathrm{IC}_{50}$ value $\left(10.25 \mu \mathrm{gmL}^{-1}\right)$ was found for $\mathrm{n}$ hexane fraction for Colo- 205, a colon cancer cell line, whereas $17.86 \mu \mathrm{g} \cdot \mathrm{mL}^{-1}$ was the highest $\mathrm{IC}_{50}$ value found for CNS cancer cell line (SF-295) ${ }^{30}$. Ma et al isolated racemosalactones A, alantolactone, isoalantolactone, alloalantolactone, 5- $\alpha$ epoxyalantolactone, $\alpha$-epoxyisoalantolactone and isotelekin from the methanol roots extract of Inula racemosa. All the isolated compounds were evaluated for their antiproliferative activities using human non-small-cell lung cancer (A-549), hepatocellular carcinoma (HepG-2) and human fibrosarcoma (HT-1080) cells using CCK-8 dye. All the tested compounds exhibited anti-proliferative activities with $\mathrm{IC}_{50}$ values ranging from 0.38 to $4.19 \mu \mathrm{gmL}^{-1}$ against human non-small-cell lung cancer. A-549, hepatocellular carcinoma HepG-2, and human fibrosarcoma HT-1080 cells. Isolated compounds alantolactone and isoalantolactone were evaluated for antiproliferative activity against human umbilical vein endothelial cells (HUVECs). IC 50 values for these two compounds were found to be 2.4 and 2.5 $\mu \mathrm{gmL}^{-1}$, respectively ${ }^{42}$.

Zhang et al isolated septuplinolide, 11- $\alpha$-13-dihydro-2- $\alpha$ hydroxy-alantolactone, 11, 13-dihydroivalin and isoalantolactone from the ethanol root extract of Inula racemosa. All the isolated compounds were evaluated for their cytotoxic activities using human lung cancer (A-549), human liver cancer (BEL-7402), human stomach cancer (BGC-823), human colon cancer (HCT-8) and human ovarian cancer (A2780) cell lines using MTT assays. All the tested compounds exhibited moderate anticancer activities ${ }^{43}$. "Macrophyllilactone $\mathrm{E}$, isoalantolactone isolated from Inula racemosa was evaluated for their anti-platelet activating factor against the release of $\beta$ glucuronidase in rat's polymorphonuclear leukocytes, whereas ginkgolide used as a positive control. For these two compounds, inhibition ratio was found to be $65.4 \%$ and $80.5 \%$ respectively, at a concentration of $10 \mu \mathrm{M}$ whereas ginkgolide produce $68.3 \%$ inhibition ${ }^{43} . " 44$

The cytotoxicity of ethanol roots extract of Inula racemosa was evaluated using the SRB (Sulphorhodamine-B) and MTT assay on normal human liver cell. CTC50 value was found to be $666.14 \pm 22.44,690.14 \pm 6.74 \mu \mathrm{g} \cdot \mathrm{mL}^{-1}$ by using MTT and SRB assay respectively in Chang liver cells (normal human liver cell) 45 .

\section{Antifungal Activity}

Isoalantolactone isolated from the methanol roots extract of Inula racemosa was evaluated for antifungal activity against the human pathogenic fungi Aspergillus flavus, Aspergillus niger, Geotrichum candidum, Candida tropicalis and Candida albicans. The tested compound inhibited the growth of Aspergillus niger, Aspergillus flavus, Geotrichum candidum, Candida albicansand Candida tropicalis with MICs values 50,

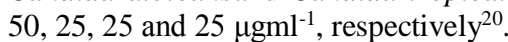




\section{Antibacterial Activity}

Antibacterial activity of the ethanol and aqueous root extract of Inula racemosa was evaluated by disc diffusion method against Escherichia coli and Staphylococcus aureus. The aqueous extract of the plant exhibited significant antimicrobial activity for these two microorganisms tested, with MIC values of 6.25 $\mathrm{mgml}^{-1}$ and $12.5 \mathrm{mgml}^{-1}$ respectively, whereas ethanol extract also had potent activity against microorganisms, with MIC of $15.625 \mathrm{mgml}^{-113}$

\section{Hepatoprotective Activity}

Hepatoprotective and curative effect of hydroalcoholic extract of the roots of Inula racemosa against hepatic ischemic/reperfusion injury in rats was examined. The plant extract at the dose of 200 and $400 \mathrm{mgkg}-1$ produced significant hepatoprotection by decreasing the elevated levels of aspartate transaminase, alanine transaminase, alkaline phosphatase and lactate dehydrogenase. It had been also seen that Inula racemosa increased the free radicals scavenging activity in the early period of hepatic ischemia/reperfusion injury in rats ${ }^{46}$. In-vitro hepatoprotective activity of ethanol roots extract of Inula racemosa was evaluated for its effect on the Chang cell line (normal human liver cells) against carbon tetrachloride induced hepatotoxicity. The cells which are exposed only with toxicant $\mathrm{CCl}_{4}$ showed $42 \%$ viability while the cells which were pretreated with extract at concentration of $600 \mu \mathrm{gmL}^{-1}$ and $300 \mu \mathrm{gmL}^{-1}$ showed an increase in percentage viability (78\%) and the results were highly significant when compared to $\mathrm{CCl}_{4}$ intoxicated cells ${ }^{13}$.

Hepatoprotective activity of isolated compound isoalantolactone was evaluated against $\mathrm{CCl}_{4}\left(2.0 \mathrm{mLkg}^{-1}\right.$ b.w. $)$ induced liver injury in male wistar rats, at a dose of $100 \mathrm{mgkg}^{-1}$ b.w. Silymarin $\left(10 \mathrm{mg} \mathrm{kg}{ }^{-1}\right)$ was used as a standard drug. The degree of protection was measured using biochemical parameters such as serum glutamate oxaloacetate transaminase (SGOT), serum glutamate pyruvate transaminase (SGPT), alkaline phosphatase (ALP) and bilirubin. The tested compound decreased the levels of these enzymes in a significant manner, similar to silymarin treated animal group when compared with $\mathrm{CCl}_{4}$ treated group ${ }^{47}$.

\section{Anti-Allergic Activity}

Mast cell stabilizing activity of $90 \%$ ethanol root extracts of Inula racemosa was evaluated on degranulation of rat peritoneal mast cell induced by compound 48/80 and egg albumin. Effect of plant extract on egg albumin induced mast cell degranulation in rats at concentration of $5,10,20$ and $40 \mu \mathrm{gmL}^{-1}$ produced dose related inhibition of $18.85,39.96,58.97$ and $71.65 \%$ respectively. Whereas, kitotifen (standard drug, $10 \mu \mathrm{gml}^{-1}$ ) was found to inhibit degranulation to avc n extent of $78.22 \%$. Effect of Inula racemosa extract on compound 48/80 induced mast cell degranulation in rats at same concentration showed reduction in degranulation to $20.36,37.08,59.52$ and $41.28 \%$ respectively while standard drug kitotifen was found to inhibit degranulation to an extent of $77.52 \%$.

"In another experiment, anti-allergic activity of alcohol extract of roots of Inula racemosa, was studied in experimental models of type-I hypersensitivity, viz. egg albumin induced passive cutaneous anaphylaxis (PCA) and mast cell degranulation in albino rats. The plant extract showed significant protection against egg albumin induced passive cutaneous anaphylaxis, both in case of single dose administration as well as with administration of extract for seven consecutive days. ${ }^{49}$
The hydroalcoholic extract of the roots of Inula racemosawas found to have potent antihistaminic activity as revealed by blockade of histamine-induced contractions of isolated tracheal chain of guinea pig"43(64). The drug also offered marked protection against bronchospasm induced by histamine, pollens of Zea maize, Holopteliasp., and Acacia arabicain guinea pigs. The beneficial effects of Inula racemosa in bronchial asthma appear to be due to its antihistaminic, anti-5-HT and antiallergic properties. ${ }^{50}$

\section{Mosquito Larvicidal Activity}

"Quinet al isolated 11, 13-dihydroisoalantolactone, macrophyllilactone E, 5- $\alpha$-epoxyalantolactone and epoxyisoalantolactone from the ethanol root extract of Inula racemosa. Mosquito larvicidal activity of all these isolated compounds was evaluated against the larvae of Aedesalbo pictus and Asian tiger mosquitoes. The tested compound 11, 13dihydroisoalantolactone and macrophyllilactone E exhibited strong larvicidal activity against the early fourth-instar larvae of Aedes albopictus with $\mathrm{LC}_{50}$ values of $21.86 \mu \mathrm{gmL}^{-1}$ and 18.65 $\mu \mathrm{gmL}^{-1}$ respectively, whereas 5- $\alpha$-epoxyalantolactone and epoxyisoalantolactone also possessed larvicidal activity against the Asian tiger mosquitoes with $\mathrm{LC}_{50}$ values of $29.37 \mu \mathrm{gmL}^{-1}$ and $35.13 \mu \mathrm{gmL}^{-1}$ respectively ${ }^{14,44}$."

\section{Antioxidant Activity}

Antioxidant activity of $70 \%$ ethanol extract of the roots of Inula racemose was performed in Albino rats. The effect of daily oral administration of alcoholic extract (suspended in $1 \%$ gum acacia) of the roots of Inula racemose to rats for 21 days was investigated for lipid peroxide formation and reduced glutathione ${ }^{51}$ content. The level of GSH in blood and liver was found significantly higher in treated animals as compared to control (1\%gum acacia). Result showed that Inula racemose has antioxidant properties because greater availability of GSH to the cell would lead to higher rate of destruction of deleterious hydrogen peroxide and lipid peroxides by glutathione peroxidase $^{13}$.

\section{Antiasthmatic Activity}

The anti-asthmatic activity of the roots extracts of Inula racemosa was evaluated by measuring the antagonistic effect on histamine-induced contraction, milk induced eosinophilia, leukocytosis and protection against mast cell degranulation in wistar rats. Petroleum ether extract the plant at a dose of 4 $\mathrm{mgmL}^{-1}(55.41 \pm 3.04)$ and $10 \mathrm{mgmL}^{-1}(48.87 \pm 1.36)$ exert significant antagonistic effect on histamine induced (1.6 $\mu \mathrm{gmL}^{-1}$ ) contraction as compared to its ethanol and aqueous extract. Milk-induced eosinophilia in mice of petroleum ether extract at a dose of $50 \& 100 \mathrm{mgkg}^{-1}$.Intraperitoneal (i.p.) was found to be $44.77 \%$ and $54.36 \%$ respectively as compared control group $(43.1 \pm 2.41)$. Similarily, dose dependent inhibition of petroleum ether extract at a dose of 50 and 100 $\mathrm{mgkg}^{-1}$, i.p. on milk induced leukocytosis $(59.53 \%$ and $77.47 \%$ ) supports the adaptogenic potential of the drug. Pretreatment with petroleum ether extract at a dose of $100 \mathrm{mgkg}^{-1}$, i.p. significantly offered protection $(74.68 \%)$ against mast cell degranulation when compared with control group ${ }^{52}$.

\section{Antimutagenic and Antiapoptotic Effects}

Protective effect of aqueous root extract of Inula racemosa was evaluated on 4-nitroquinoline-1-oxide -induced DNA damage and apoptosis in mice bone marrow cells. Aqueous root extract of Inula racemosa (100, 200 and $400 \mathrm{mgkg}^{-1}$, b.w.) with or 
without treatment with 4-nitroquinoline-1-oxide (4-NQO) were administered orally for five consecutive days. Antiapoptotic effect of aqueous root extract of I. racemosa $\left(400 \mathrm{mgkg}^{-1}\right.$, b.w.) was measured by the use of Annexin V-FITC assay kit. 4-NQOinduced genetic damage in mice was modulated by aqueous root extract of Inula racemosa via effective restoration of micronuclei and apoptotic cells formations. The potential protective effects might be due to the synergistic effects of secondary metabolites present in aqueous root extract of Inula racemosa $a^{53}$

\section{Adaptogenic Activity}

Adaptogenicity potential of $90 \%$ ethanol roots extract of Inula racemosa was investigated in the forced swim test model in albino mice. The animals treated with $100 \mathrm{mgkg}^{-1}$ and 200 $\mathrm{mgkg}^{-1}$ of ethanol root extract of Inula racemosa showed a significant decrease in the immobility period with simultaneous increase in antioxidant markers, adrenaline and serotonin levels ${ }^{45}$.

\section{Adrenergic $\beta$-Receptor Blocking Activity}

"The adrenergic $\beta$-receptor blocking activity of the petroleum ether extract of the roots of Inula racemosa was evaluated in rats. The plant extract showed lowered plasma insulin and glucose levels within $75 \mathrm{~min}$ of oral administration and it significantly neutralized adrenaline induced hyperglycaemia. Furthermore, the extract showed negative ionotropic and negative chronotropic effects on frog heart. These findings suggest that Inula racemosa exhibited $\beta$-receptor blocking activity ${ }^{11}$.

\section{Hypoglycemic Activity}

Endocrine response of ethanol roots extract of Inula racemosa was evaluated in relation to glucose homeostasis in rats. It was found that alcoholic extract of the roots of Inula racemosa lowers blood glucose level and enhances liver glycogen without increasing plasma insulin level in rats ${ }^{54}$.

Anti-diabetic effect of Inula racemosa roots powder was performed in 15 patients of age above 35 years suffered from the complications of diabetes mellitus like polyurea; polydypsia and polyphagia etc. were selected for the clinical study. All the patients were treated with 1 tablespoonful of Inula racemosa roots powder three times in a day for three months duration. The response was estimated on the parameter of Joslin's Clinica. After the treatment blood glucose level of all patients was found to be normal ${ }^{55}$,"

Roots of Inula racemosa were evaluated for the amelioration of corticosteroid (dexamethasone) induced hyperglycaemia in mice. Corticosteroid administration in the animals increased the serum glucose level. Roots of Inula racemosa decreased the serum concentrations of the thyroid hormones tetraiodothyronine (T4) and triiodothyronine (T3) in corticosteroid-induced hyperglycaemic mice which was found comparable with standard drug ketoconazole. Findings of the results suggest that hypoglycemic effect of the extract was mediated through its cortisol inhibiting potency ${ }^{10,56}$. Ethanol extract of the roots of Inula racemosa was evaluated for the effect on glucose metabolism in albino rats. Blood glucose, plasma insulin and liver glycogen levels were measured after 2, $4,8,16$ and 24 hours of drug administration. At a dose of 400 $\mathrm{mg} \mathrm{kg}{ }^{-1}$, b.w. plasma glucose level decreased after 4 hours of drug administration and returned to normal at 16 hours. Liver glycogen level was increased significantly as compared to control group at 4 hours after drug administration. A significant reduction in plasma insulin level was observed 4 hours after drug administration, and returned to normal at 8 hour, and remained low up to 16 hours ${ }^{57}$.

Water decoction of the root of Inula racemosa has been reported not only to lower the fasting blood glucose in normal rabbits, but also to protect the rabbit against glucose included hyperglycemia ${ }^{58}$. Chronic treatment with methanol root extract of Inula racemosa produced significant reduction in blood sugar level in alloxan-induced hyperglycemia model as compared to alloxan treated animals. The body weight, food intake, water intake and urine output were significantly reversed to normal by methanol extract of Inula racemosa treatment ${ }^{59}$.

\section{Cardioprotective Activity}

The cardioprotective potential of hydroalcohol extract of roots of Inula racemosa was evaluated against isoproterenol-induced myocardial infarction in rats. The rats were treated with isoproterenol $\left(85 \mathrm{mgkg}^{-1}\right.$, subcutaneous) exhibited myocardial infarction, like decrease in arterial pressure, heart rate, contractility, relaxation along with increased left ventricular end diastolic pressure, as well as decreased endogenous myocardial enzymatic and non-enzymatic antioxidants. Isoproterenol also significantly induced lipid peroxidation and increased leakage of myocyte injury marker enzymes. Pretreatment with Inula racemosa extract (100 and $200 \mathrm{mg} \mathrm{kg}^{-1}$ per day, per oral) for 21 consecutive days, significantly restored the reduced form of glutathione and endogenous antioxidant enzymes superoxide dismutase, catalase, glutathione peroxidase from the heart, which were depleted after isoproterenol administration ${ }^{60}$.

In another experiment it has been found that ethanol root extract of Inula racemosa possess cardioprotective activity against isoproterenol induced myocardial infarction treated wistar rats by restoring electrocardiographic, histopathological and biochemical changes. Myocardial infarction was induced in the wistar rats by isoproterenol administration (200 $\mathrm{mgkg}^{-1}$ subcutaneously twice at an interval of $24 \mathrm{~h}$ ). Ethanol roots extract of I. racemosa markedly restrained isoproterenolinduced electrocardiographic changes indicative of its cell membrane protecting effects. At a dose of 400, 600 and $800 \mathrm{mg}$ $\mathrm{kg}^{-1}$ daily for a period of 10 days, it improved cardiac function, decreased oxidative stress, cardiac injury and maintained cell membrane integrity and lipid peroxidation process in a dose dependent manner. In addition, it has normalized histopathological changes caused by isoproterenol administration ${ }^{61}$.

In another experiment myocardial ischemia was induced in rats by isoproterenol administration $\left(20 \mathrm{mg} 100 \mathrm{~g}^{-1}\right.$ subcutaneously twice at an interval of $24 \mathrm{~h}$ ). The petroleum ether extract of roots of the plant Inula racemosa and alantolactone, which have been isolated from the roots of the plant were subjected for evaluation of their cardioprotective activity in myocardial ischemia. Lipid peroxides and glutathione contents were anticipated. It has been found that the alantolactone as well as petroleum ether extract effectively reduces the lipid peroxide levels in the ischemic rats and brings the glutathione content to near normal level ${ }^{62}$.

A combination of the plant Commiphora mukul and Inula racemosa in 1:1 ratio was studied in 200 patients suffered with ischemic heart disease. The major symptoms included chest pain, with ST-segment and T-wave changes on the electrocardiogram (ECG), suggested myocardial ischemia in about 80 percent of the patients. Pretreatment with combination of the plant Commiphora mukul and Inula racemosa in 1:1 ratio 
to the patients caused improvement in precordial pain and dyspnea, restoration of normal ECG patterns, and significant reductions in cholesterol, triglycerides and total lipid levels ${ }^{63}$.

The isolated compound from Inula racemosa was evaluated for the cardioprotective activity on isolated frog heart at a dose 40 $\mu \mathrm{gmL}^{-1}$ showed that alantolactone decreased heart rate and force of contraction. The study indicated that the alantolactone produces a negative ionotropic and negative chronotropic effect on frog's heart ${ }^{64}$. Cardioprotective activity of ethanol root extract of Inula racemosawas also evaluated in wistar male albino rats having myocardial ischemic reperfusion injury. "The extract at a dose of $100 \mathrm{mgkg}^{-1}$ for 30 days appreciably restored the myocardial antioxidant status evidence by increased superoxide dismutase (SOD), catalase (CAT), glutathione peroxidase (GPx), reduced glutathione ${ }^{51}$ and prevented leakage of cardiomyocytes specific enzymes, creatine phosphokinase isoenzyme and lactate dehydrogenase $(\mathrm{LDH})$. The result suggested cardioprotective effect of Inula racemosalikely resulted to improve antioxidant status, haemodynamic and left ventricular contractile function subsequent to suppression of oxidative stress". 60

\section{DISCUSSION}

Many of the local and traditional claims for the use of different parts of Inula racemosa have been scientifically established by in vivo and in vitro techniques. Sesquiterpene lactones have received considerable attention because of their numerous biological activities ${ }^{28}$. In addition, these compounds exert their allelopathic effects on other flora and vertebrate poisoning ${ }^{29}$. The plant is used in Ayurveda as an expectorant and resolvent in indurations. Considered a rejuvenator and immunomodulator by Ayurvedic physicians, the drug according to Bhavaprakasha ${ }^{65}$ is bitter pungent in taste. When administered it mitigates Vatakapha Jwara (fever caused by vata pitta imbalance), sotha (swelling), aruchi (anorexia), swasa (breathlessness) and parswasoola (pain in the sides of the chest)". The root of Inula racemosa is an important ingredient of several polyherbal formulations those are for cardiac disease and inflammatory conditions of spleen and liver. Besides compounds of Inula racemosa root and Comiphora mukul called Pushkar Guggulu is a popular anti obesity, hypolipidemic is indicated in cardiac ailments.

The root is medicinal and considered a specific for cough, dyspnea, asthma, pleurisy, tuberculosis and myocardial ischemia and chest pain especially pre cordial pain. Root powder is reportedly hypoglycemic and hypocholesterolemic in human subjects ${ }^{14}$. It brought about a beneficial improvement in ST-T changes in ECG of patients with Ischemic heart disease (IHD).

The aqueous extract of the fresh or dry roots is given orally in rheumatic pains and liver problems. Externally a paste or liniment is used for relieving pain. The root is also used in veterinary medicine as a tonic. The root forms an important ingredient of several polyherbal formulations for heart diseases and inflammatory conditions of spleen and liver. Along with Commiphora mukul, the drug combination called 'Pushkarguggulu' is a popular anti obesity, hypolipidemic indicated in cardiac ailments. Inula racemosa is used in Chinese medicine for abdominal distension and pain, acute enteritis and bacillary dysentery" 43

\section{CONCLUSION}

Inula racemosa is a medicinal plant of immense importance with a diverse pharmacological spectrum. There is a great scope for further screening of the plant against various respiratory, antihistaminic and cardiovascular disorders. The plant can also be evaluated against hyperglycemia, cytoprotective and hepatoprotective properties. Thus the broad spectrum of the biological activities of alantolactone and isoalantolactone completely justifies the Russian name of the compound from which these compounds were isolated - devyasil (nine powers). Moreover, the phytochemical screening can also be performed to explore new chemical entities present in the plant for further exploitation of species.

\section{REFERENCES}

1. Singh, G.; Paul, V.; Handa, K., Chemical composition of the essential oil of Inula racemosa roots from plants growing in Jammu and Kashmir. J. Sci. Indust. Res. B 1959, 18, 351-352.

2. Chopra, R. N.; Nayar, S. L.; Chopra, I. C., Glossary of Indian medicinal plants. New Delhi.: C SIR 1956.

3. Singh, R.; Singh, R.; Batliwala, P.; Upadhyay, B.; Tripathi, S., Puskara-Guggulu, an Anti-anginal and Hypolipidemic Agent in Coronary Heart Diseases (CHD). Jour. Res. Ay. \& Siddha 1991,12.

4. Arora, R.; Maheshwari, M.; Chandel, K.; Gupta, R., Mano (Inula racemosa): Little known aromatic plant of Lahaul valley, India. Economic Botany 1980,34 (2), 175-180.

5. Milman, I., Alanto-and isoalantolactones. Chemistry of Natural Compounds 1990,26 (3), 251-262.

6. Moerman, D. E. Medicinal plants of native America; 1986.

7. Tripathi, S.; Tiwari, C.; Upadhyay, B.; Singh, R., Screening of hypoglycemic action in certain indigenous drugs. Journal of Research in Indian Medicine, Yoga and Homeopathy 1979,14, 159-169.

8. Tripathi, S.; Upadhyaya, B.; Gupta, V., Beneficial effect of Inula racemosa (pushkarmoola) in angina pectoris: a preliminary report. Indian journal of physiology and pharmacology 1983,28 (1), 73-75.

9. Seth, S.; Maulik, M.; Katiyar, C.; Maulik, S., Role of Lipistat in Protection against Isoproterenol Induced Myocardial Necrosis in Rats: A Biochemcial and Histopathological Study. Indian journal of physiology and pharmacology 1998,42, 101-106.

10. Gholap, S.; Kar, A., Effects of Inula racemosa root and Gymnema sylvestre leaf extracts in the regulation of corticosteroid induced diabetes mellitus: involvement of thyroid hormones. Die Pharmazie-An International Journal of Pharmaceutical Sciences 2003,58 (6), 413-415.

11. Tripathi, Y.; Tripathi, P.; Upadhyay, B., Assessment of the adrenergic beta-blocking activity of Inula racemosa. Journal of ethnopharmacology 1988,23 (1), 3-9.

12. Rao, K.; Mishra, S., Hepatoprotective activity of Inuta racemosa root. Fitoterapia 1997,68 (6), 510-514.

13. Sharma, V.; Hem, K.; Sharma, D.; Singh, V. P.; Singh, N. K., Journal of Natural Products and Resources.

14. Tripathi, V.; Agarwal, S.; Srivastava, O.; Rastogi, R., Antidermatophytic constituents from Inula racemosa Hook f. Indian Journal of Pharmaceutical Sciences 1978,40 (4), 129-131.

15. Raghavan, R.; Ravindranath, K.; Trivedi, G.; Paknikar, S.; Bhattacharyya, S., Inunolide--a new sesquiterpene lactone from Innula racemosa root. Indian journal of chemistry 1969.

16. Ravindranath, K.; Raghavan, R.; Paknikar, S.; Trivedi, G.; Bhattacharyya, S., Structure and stereochemistry of inunolide, dihydroinunolide and neoalantolactone. 1978.

17. Bhandari, P.; Rastogi, R., Alloalantolactone, a sesquiterpene lactone from inula-racemosa. Council scientific industrial research publ \& info directorate, new delhi 110012, india: 1983; Vol. 22, p. 286-287. 
18. Kalsi, P.; Goyal, R.; Talwar, K.; Chhabra, B., Epoxy alantolides: isoinunal - a new potent plant growth regulator from Inula racemosa. Phytochemistry 1988,27 (7), 2079 2081.

19. Kalsi, S.; Goyal, R.; Talwar, K.; Chhabra, B., Stereostructures of two biologically active sesquiterpene lactones from Inula racemosa. Phytochemistry 1989,28 (8), 2093-2096.

20. Tan, R.; Tang, H.; Hu, J.; Shuai, B., Lignans and sesquiterpene lactones from Artemisia sieversiana and Inula racemosa. Phytochemistry 1998,49 (1), 157-161.

21. Matthews, V.; Åström, M.-B.; Chan, M.; Bruce, C.; Krabbe, K.; Prelovsek, O.; Åkerström, T.; Yfanti, C.; Broholm, C.; Mortensen, O., Brain-derived neurotrophic factor is produced by skeletal muscle cells in response to contraction and enhances fat oxidation via activation of AMP-activated protein kinase. Diabetologia 2009,52 (7), 1409-1418.

22. Ober, A. G.; Fischer, N. H., Sesquiterpene lactones from Calea septuplinervia. Phytochemistry 1987,26 (3), 848-849.

23. Fu, B.; Su, B.-N.; Takaishi, Y.; Honda, G.; Ito, M.; Takeda, Y.; Kodzhimatov, O. K.; Ashurmetov, O., A bissesquiterpene and sesquiterpenolides from Inula macrophylla. Phytochemistry 2001,58 (7), 1121-1128.

24. Kashman, Y.; Lavie, D.; Glotter, E., Sesquiterpene lactones from Inula helenium. Israel Journal of Chemistry 1967,5 (1), 23-27.

25. Al-Yahya, M. A.; Khafagy, S.; Shihata, A.; Kozlowski, J. F.; Antoun, M. D.; Cassady, J. M., Phytochemical and biological screening of Saudi medicinal plants, Part 6 . Isolation of $2 \alpha$-hydroxyalantolactone the antileukemic principle of Francoeuria crispa. Journal of natural products 1984,47 (6), 1013-1017.

26. Topçu, G.; Öksüz, S.; Shieh, H.-L.; Cordell, G. A.; Pezzuto, J. M.; Bozok-Johansson, C., Cytotoxic and antibacterial sesquiterpenes from Inula graveolens. Phytochemistry 1993,33 (2), 407-410.

27. Kaur, B.; Kalsi, P., Stereostructures of inunal and isoalloalantolactone, two biologically active sesquiterpene lactones from Inula racemosa. Phytochemistry 1985,24 (9), 2007-2010.

28. Picman, A. K., Biological activities of sesquiterpene lactones. Biochemical Systematics and Ecology 1986,14 (3), 255-281.

29. Vidari, G.; De Bernardi, M.; Vita-Finzi, P.; Fronza, G., Sesquiterpenes from Lactarius blennius. Phytochemistry 1976,15 (12), 1953-1955.

30. Pal, H. C.; Sehar, I.; Bhushan, S.; Gupta, B. D.; Saxena, A. $\mathrm{K}$., Activation of caspases and poly (ADP-ribose) polymerase cleavage to induce apoptosis in leukemia HL60 cells by Inula racemosa. Toxicology in vitro 2010,24 (6), 1599-1609.

31. Dalvi, R.; Singh, B.; Salunkhe, D. K., A study on phytotoxicity of alantolactone. Chemico-biological interactions 1971,3 (1), 13-18.

32. Jagdale, A. C. S. C., Antibacterial activity of isolated constituents and extract of roots of Inula racemosa. Research Journal of Medicinal Plant 2007,1 (1), 7-12.

33. Xu, R.; Zhou, G.; Peng, Y.; Wang, M.; Li, X. Pharmacokinetics, tissue distribution and excretion of isoalantolactone and alantolactone in rats after oral administration of Radix Inulae extract. Molecules 2015,20 (5), 7719-7736.

34. Ohta, Y.; Andersen, N. H.; Liu, C.-B., Sesquiterpene constituents of two liverworts of genus Diplophyllum: novel eudesmanolides and cytotoxicity studies for enantiomeric methylene lactones. Tetrahedron 1977,33 (6), $617-628$
35. Woerdenbag, H.; Meijer, C.; Mulder, N.; De Vries, E.; Hendriks, H.; Malingré, T. M., Evaluation of the in vitro cytotoxicity of some sesquiterpene lactones on a human lung carcinoma cell line using the fast green dye exclusion assay. Planta medica 1986,52 (02), 112-114.

36. Kupchan, S. M.; Eakin, M.; Thomas, A., Tumor inhibitors. 69. Structure-cytotoxicity relations among the sesquiterpene lactones. Journal of Medicinal Chemistry 1971,14 (12), 1147-1152.

37. Abeysekera, B.; Abramowski, Z.; Towers, G., Chromosomal aberrations caused by sesquiterpene lactones in chinese hamster ovary cells. Biochemical systematics and ecology 1985,13 (3), 365-369.

38. Dupuis, G.; Brisson, J., Toxic effect of alantolactone and dihydroalantolactone in in vitro cultures of leukocytes. Chemico-biological interactions 1976,15 (3), 205-217.

39. Khan, A.; Shah, R.; Pallewar, S., Evaluation of Antiinflammatory and Analgesic Activity of Ethanolic extracts of Inula racemosa and Albizia amara. Int $\mathbf{J}$ Pharmacog Phytochem Res 2010,3, 22-27.

40. Fallahzadeh, A.; Mohammadi, S., An Investigation of the Antinociceptive and Anti-inflammatory Effects of Hydroalcoholic Extract of Inula Helenium on Male Rats. Journal of Babol University of Medical Sciences 2016,18 (12), 57-63.

41. Arumugam, P.; Murugan, M.; Thangaraj, N., Evaluation of anti-inflammatory and analgesic effects of aqueous extract obtained from root powder of Inula racemosa Hook. f. Journal of Medicinal Plants Research 2012,6 (14), 28012806.

42. Ma, Y.-Y.; Zhao, D.-G.; Gao, K., Structural investigation and biological activity of sesquiterpene lactones from the traditional Chinese herb Inula racemosa. Journal of natural products 2013,76 (4), 564-570.

43. Zhang, T.; Xiao, W.; Gong, T.; Yang, Y.; Chen, R.-Y.; Yu, D.-Q., Two new eudesmanolides from Inula racemosa. Journal of Asian natural products research 2010,12 (9), 788-792.

44. He, Q.; Liu, X. C.; Sun, R. Q.; Deng, Z. W.; Du, S. S.; Liu, Z. L., Mosquito larvicidal constituents from the ethanol extract of Inula racemosa Hook. f. Roots against Aedes albopictus. Journal of Chemistry 2014,2014.

45. Gnanasekaran, D.; Reddy, C.; Jaiprakash, B.; Narayanan, N.; Kiran, Y.; Elizabeth, H., Adaptogenic activity of siddha medicinal plant Inula racemosa. Int. J. Biol. Pharm. Allied Sci 2012,1 (6), 870-880.

46. Manipuri, P.; Indala, R.; Jagaralmudi, A.; Ramesh Kumar, K., Hepatoprotective Effect of Inula racemosa on Hepatic Ischemia/reperfusion Induced Injury in Rats. J Bioanal Biomed 2013,5, 022-027.

47. Kalachaveedu, M.; Kuruvilla, S.; Kedike, B., Hepatoprotective activity of isoalantolactone isolated from the roots of Inula racemosa (Hook. F.). Indian Journal of Natural Products 2015,29 (1), 56.

48. Choudhary, G., Mast cell stabilizing activity of Inula racemosa linn. Int. J. Res. Rev. Pharm. Appl. Sci 2012,2 (4), 630-636.

49. Srivastava, S.; Gupta, P.; Prasad, R.; Dixit, K.; Palit, G.; Ali, B.; Misra, G.; Sexena, R., Evaluation of Antiallergic Activity (Type I Hypersensitivity) of lnula Racemosa in Rata. Indian journal of physiology and pharmacology 1999,43, 235-241.

50. Singh, N.; Nath, R.; Gupta, M.; Kohli, R., An Experimental Evaluation of Anti-Asthmatic Potentialitis of Inula racemosa (Puskar Mul). Quarterly Journal of Crude Drug Research 1980,18 (2), 89-96.

51. Wei, P.; Liu, Q.; Li, D.; Zheng, Q.; Zhou, J.; Li, J., Acute nicotine treatment attenuates lipopolysaccharide-induced 
cognitive dysfunction by increasing BDNF expression and inhibiting neuroinflammation in the rat hippocampus. Neuroscience letters 2015,604, 161-166.

52. Vadnere, G. P.; Gaud, R. S.; Singhai, A. K.; Somani, R., Effect of Inula racemosa root extract on various aspects of asthma. Pharmacologyonline 2009,2, 84-94.

53. Arumugam, P.; Murugan, M., Antimutagenic and antiapoptotic effects of aqueous root extract of Inula racemosa Hook. f. on 4-NQO-induced genetic damage in mice. ISRN pharmacology 2013,2013.

54. Tripathi, Y.; Chaturvedi, P., Assessment of endocrine response of Inula racemosa in relation to glucose homeostasis in rats. Indian journal of experimental biology 1995,33 (9), 686-689.

55. Singh, T.; Upadhyay, B.; Tewari, C.; Tripathi, S., Management of diabetes mellitus (prameha) with Inula racemosa and Cinnamomum tamala. Ancient science of life 1985,5 (1), 9.

56. Gholap, S.; Kar, A., Hypoglycaemic effects of some plant extracts are possibly mediated through inhibition in corticosteroid concentration. Die Pharmazie-An International Journal of Pharmaceutical Sciences 2004,59 (11), 876-878.

57. Chaturvedi, P.; Shukla, S.; Tripathi, P.; Chaurasia, S.; Singh, S.; Tripathi, Y., Comparative study of Inula Racemosa and Saussurea Lappa on the glucose level in Albino rats. Ancient science of life 1995,15 (1), 62.

58. Singh, N.; Nath, R.; Tripathi, S.; Sharma, V.; Kohli, R., Pharmacological studies on Inula racemosa Hook. J. Res. Indian Med 1976.

59. Ajani, H.; Patel, H.; Shah, G.; Acharya, S.; Shah, S., Evaluation of antidiabetic effect of methanolic extract of
Inula racemosa root in rats. Pharmacologyonline 2009,3, 118-129.

60. Ojha, S.; Nandave, M.; Kumari, S.; Arya, D. S., Cardioprotection by Inula racemosa Hook in experimental model of myocardial ischemic reperfusion injury. 2010.

61. Shirole, T.; Jagtap, A.; Phadke, A.; Velhankar, R., Preventive effect of ethanolic extract of Inula racemosa on electrocardiograph-ic, biochemical and histopathological alterations in isoproterenol-induced myocardial infarction in rats. Int. J. Res. Phytochem. Pharmacol 2013,3 (1), 13 22.

62. Chabukswar, A.; Kuchekar, B.; Jagdale, S.; Lokh, P.; Raut, C., Cardio protective activity of Inula racemosa. International Journal of Chemical Sciences 2010,8 (3).

63. Singh, R.; Singh, R.; Ram, P.; Batliwala, P., Use of Pushkar-Guggul, an indigenous antiischemic combination, in the management of ischemic heart disease. International journal of pharmacognosy 1993,31 (2), 147-160.

64. Lokhande, P.; Dhaware, B.; Jagdale, S.; Chabukswar, A.; Mulkalwar, S., Cardiac activity of isolated constituents of Inula racemosa. Journal of herbal pharmacotherapy 2006,6 (3-4), 81-88.

65. Bhattacharyya, C., Therapeutic potential of Giloe Tinospora cordifolia The Magical Herb of Ayurveda. International Journal of Pharmaceutical \& Biological Archive 2013,4 (4).

\section{Cite this article as:}

Qurba Firdous et al. Ethnopharmacology, phytochemistry and biological activity of Inula racemosa Hook. F: A review. Int. J. Res. Ayurveda Pharm. 2018;9(1):95-102 http://dx.doi.org /10.7897/2277-4343.09120

Disclaimer: IJRAP is solely owned by Moksha Publishing House - A non-profit publishing house, dedicated to publish quality research, while every effort has been taken to verify the accuracy of the content published in our Journal. IJRAP cannot accept any responsib ility or liability for the site content and articles published. The views expressed in articles by our contributing authors are not necessarily those of IJRAP editor or editorial board members. 